\title{
Long-term follow-up from PROTECT AF trial reported
}

Atrial fibrillation is associated with an increased risk of stroke. Thromboembolisms that cause stroke in patients with atrial fibrillation are hypothesized to come from the left atrial appendage (LAA). The results of the PROTECT AF study, in which LAA closure with the Watchman ${ }^{\circledR}$ device (Atritech, Inc., Plymouth, MN, USA) was compared with standard medical therapy, now show that LAA closure is noninferior to warfarin treatment for stroke prevention in patients with atrial fibrillation.

The PROTECT AF researchers recruited 707 patients with atrial fibrillation and at least one other risk factor for stroke, and randomly assigned them to receive either LAA closure $(n=463)$ or continued warfarin therapy $(n=244)$. The rate of the primary end point-a composite of stroke, systemic embolism, and cardiovascular death-was 3.0\% and 4.3\% per 100 patient-years in the LAA closure and warfarin groups, respectively (rate ratio $0.71,95 \%$ CI $0.44-1.30 \%$ per year).

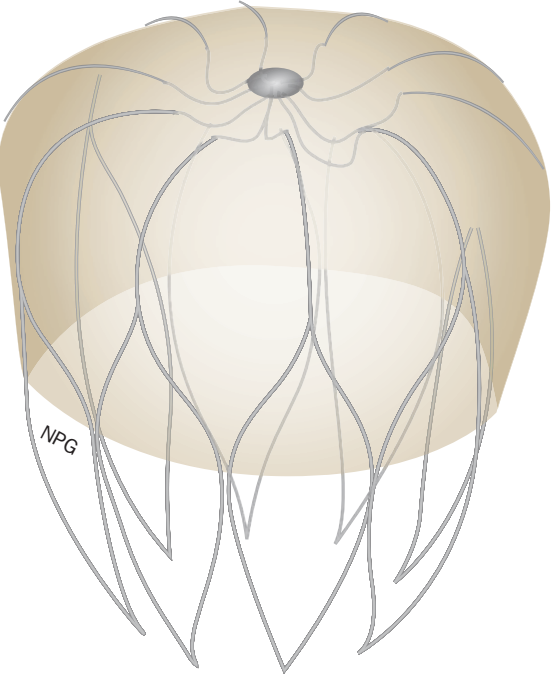

LAA closure did not achieve superiority over warfarin because of the acute, procedure-related stroke events and pericardial tamponade seen in the LAA closure group. Although most patient safety events in the LAA closure group happened during or immediately after the intervention, even after 1,588 patient-years (mean follow-up 2.3 years), more primary safety events occurred in the LAA closure group (5.5\% per year, 95\% CI $4.2-7.1 \%$ per year) than in the warfarin group $(3.6 \%$ per year, 95\% CI 2.2-5.3\% per year).

Patients receiving LAA closure were treated with warfarin for 6 weeks, then clopidogrel (antiplatelet therapy) for 4.5 months after device implantation. Secondary analysis to isolate successful device deployment from the confounding effects of drug treatment demonstrated that LAA closure is superior to warfarin treatment when events occurring on the day of device deployment are excluded. Further studies will determine the longterm safety and efficacy of LAA closure, and could identify a subgroup of patients who benefit most from this procedure.

Megan Cully

Original article Reddy, V. K. et al. Percutaneous left atrial appendage closure for stroke prophylaxis in patients with atrial fibrillation: 2.3 year follow-up of the PROTECT AF trial. Circulation doi:10.1161/CIRCULATIONAHA.112.114389 